\title{
Loss of treatment benefit due to low compliance with bisphosphonate therapy
}

\author{
F. J. A. Penning-van Beest • J. A. Erkens • M. Olson • \\ R. M. C. Herings
}

Received: 26 March 2007 / Accepted: 22 August 2007 /Published online: 14 September 2007

(C) International Osteoporosis Foundation and National Osteoporosis Foundation 2007

\begin{abstract}
Summary Among 8,822 new female bisphosphonate users, non-compliant bisphosphonate use was associated with a $45 \%$ increased risk of osteoporotic fracture compared to compliant use (MPR $\geq 80 \%$ ). Classifying compliance into five categories, fracture risk gradually increased with poorer compliance. These results emphasize the importance of treatment compliance in obtaining maximal treatment benefit.

Introduction Bisphosphonates are widely used to treat osteoporosis and reduce fracture risk. Low compliance is frequent and will limit treatment benefit.

Methods New female users of alendronate or risedronate between 1999-2004, aged $\geq 45$ years were identified from PHARMO-RLS, including drug-dispensing and hospitalization data of $\geq 2$ million residents of the Netherlands. Patients were followed until first hospitalisation for an osteoporotic fracture, death, or end of study period. Compliance with bisphosphonates during follow-up was measured over 90-day intervals using Medication Possession Ratio (MPR). The association between compliance and fracture risk was analyzed using time-dependent Cox-regression.
\end{abstract}

The study was financially supported by an unrestricted grant from Novartis Pharma AG, Basel, Switzerland.

F. J. A. Penning-van Beest $(\bowtie) \cdot J$. A. Erkens $\cdot$ R. M. C. Herings PHARMO Institute,

P.O.Box 85222, 3508 AE Utrecht, The Netherlands

e-mail: fernie.penning@pharmo.nl

M. Olson

Novartis Pharma AG,

Basel, Switzerland

R. M. C. Herings

Department of Health Policy \& Management,

Erasmus Medical Center,

Rotterdam, The Netherlands
Results The study cohort included 8,822 new female bisphosphonate users, contributing in total 22,484 person-years of follow-up. During follow-up, 176 osteoporotic fractures occurred (excluding the first six months). Non-compliant bisphosphonate use was associated with a $45 \%$ increased fracture risk compared to compliant use (MPR $\geq 80 \%$ ). Classifying compliance into five categories, fracture risk gradually increased with poorer compliance ( $\mathrm{p}$-value $<0.05$ for trend). A MPR $<20 \%$ was associated with an $80 \%$ increased fracture risk compared to a MPR $\geq 90 \%$.

Conclusions These results show a statistically significant association between level of compliance with bisphosphonates and level of fracture risk, emphasizing the importance of treatment compliance in obtaining maximal treatment benefit.

Keywords benefit $\cdot$ bisphosphonates $\cdot$ fracture . non-compliance $\cdot$ osteoporosis

\section{Introduction}

Osteoporosis is a systemic condition characterized by low bone mass and microarchitectural deterioration of bone tissue, leading to enhanced bone fragility and, consequently, an increased fracture risk [1]. Osteoporosis has clinical and public health importance because osteoporotic fractures are one of the most common causes of disability and a major contributor to medical costs in many regions of the world [2]. Bisphosphonates are potent inhibitors of the osteoclast-mediated resorption of bone and widely used to treat osteoporosis and reduce the risk of osteoporotic fractures [3].

Long-term therapy with bisphosphonates is required to realise the full benefits of this treatment [3, 4]. This is 
supported by a recent PHARMO study that showed that the risk of fractures was reduced by $26 \%$ after one year persistent bisphosphonate use and up to $32 \%$ after two year persistent use [5]. However, in daily clinical practice, $50 \%$ to $75 \%$ of patients discontinue bisphosphonate use already in the first year of therapy [5-8]. Moreover, noncompliance with bisphosphonates has also been reported to be a frequent issue, with rates varying from $35 \%$ to $65 \%$ $[6,9]$. Reasons for low compliance with bisphosphonate treatment are the stringent regimen to minimize the risk of oesophageal irritation, the existence of drug-related gastrointestinal side effects, and the fact that osteoporosis is often asymptomatic in early stages [10]. Caro et al. showed that patients who were compliant with their osteoporosis medication, including bisphosphonates, estrogens and calcitonin, experienced a $16 \%$ lower fracture rate compared to non-compliant users [11]. The objective of this study was to investigate the risk of osteoporotic fractures associated with low compliance with bisphosphonates in more detail. In addition to dichotomizing compliance, we also classified compliance into five categories.

\section{Patients and methods}

\section{Setting}

Data were obtained from the PHARMO Record Linkage System (PHARMO RLS) which includes several databases, among which drug dispensing and hospitalization data of more than two million residents of the Netherlands. The drug dispensing histories contain data on the dispensed drug, the type of prescriber, the dispensing date, the amount dispensed, the prescribed dose regimens, and the duration of use. All drugs are coded according to the Anatomical Therapeutic Chemical (ATC) Classification. The hospital records include detailed information concerning the primary and secondary diagnoses, procedures, and dates of hospital admission and discharge. All diagnoses are coded according to the International Classification of Diseases, 9th Revision, Clinical Modification (ICD-9-CM).

\section{Study cohort}

The source population included all new users of bisphosphonates in the period from January 1999 until July 2004. These patients were not dispensed any anti-osteoporotic drugs (bisphosphonates, raloxifene, tibolone, estrogens, hormone replacement therapy, calcitonin or teriparatide) for at least one year before their first bisphosphonate dispensing. All female new users of alendronate $(10 \mathrm{mg}$ daily or $70 \mathrm{mg}$ weekly) or risedronate (5 $\mathrm{mg}$ daily or $35 \mathrm{mg}$ weekly) (i.e. the dosages indicated for the prevention or treatment of postmenopausal osteoporosis), aged $\geq 45$ years or with diagnosed postmenopausal osteoporosis (ICD-9-CM code 733.01), without gaps in registration in PHARMO RLS, and with a registration of at least one year before and one year after starting bisphosphonate treatment were included in the study cohort.

All study patients were followed from the first bisphosphonate dispensing until the occurrence of the outcome of interest, death, end of registration in PHARMO RLS or end of the study period, whichever event was earliest.

\section{Compliance}

Compliance with bisphosphonates during follow-up may change and was measured over 90-day intervals using the Medication Possession Ratio (MPR). MPR was defined as the sum of days' supply of all alendronate and risedronate dispensings during or overlapping the 90-day interval divided by 90 potential days of bisphosphonate therapy. For each dispensing only the days' supply covering a specific interval were counted. E.g. of a 100-days supply dispensed at follow-up day 60 , only 30 days were counted when calculating the MPR over the first interval (day 0-90) and 70 days for the MPR over the second interval (day 91-180). The maximum value of a MPR was set at 1 . Subdividing follow-up in larger intervals was assumed too rough and subdividing in smaller intervals was not feasible regarding the usual prescription length of 90 days.

\section{Osteoporotic fractures}

The outcome variable osteoporotic fracture was defined as hospitalisation for an osteoporotic fracture during followup. Hospitalisations were selected based on primary discharge diagnosis for probable (ICD-9-CM codes 805.2 (vertebral thoracic), 805.4 (vertebral lumbar), 820 (proximal femur), 812 (proximal humerus), 813.4 (distal radius/ulna), 814 (carpus)) or possible (ICD-9-CM codes 823.0 (proximal tibia/fibula), 807.0 (rib), 807.2 (sternum), 808.0 (pelvis)) osteoporotic fractures.

\section{Covariates}

For all patients, a history of osteoporotic fracture was assessed. Furthermore, we determined use of non-steroidal anti-inflammatory drugs, analgesics, benzodiazepines and antidepressants in the year after starting bisphosphonate treatment as these drugs are associated with an increased risk of fractures [12-14]. Similarly, we assessed use of thiazide diuretics, $\beta$-blockers and statins in the year after start as these drugs are associated with a reduced risk of fractures [15-17]. 
Statistical analyses

The association between low compliance with bisphosphonates and the risk of fractures was analysed univariately and multivariately using time-dependent Cox regression analysis to account for changing compliance over time. In this analysis, at the time of each fracture, the cumulative compliance up to and including the corresponding 90-day interval of women who had experienced a fracture was compared to the cumulative compliance of those women who remained fracture-free at this time. The cumulative compliance of the fracture-free patients was measured up to the interval of corresponding fracture.

In a first analysis, compliance was dichotomized and a MPR $<80 \%$, i.e. non-compliant bisphosphonate use was compared to a MPR $\geq 80 \%$, i.e. compliant use. In a second analysis, compliance was classified into five categories: $<20 \%, 20-49 \%, 50-69 \%, 70-89 \%$ and $\geq 90 \%$ (reference). A Wald test for trend was performed. Assuming that the effect of bisphosphonates is not immediate, and in line with Caro et al. [11], fractures occurring in the first 182 days of follow-up were excluded from the analyses. In a subanalysis, this exclusion period was varied. Furthermore, an additional analysis was performed excluding women with only one bisphoshonate dispensing during follow-up; in these patients it is less sure that they actually used the drug.

The multiple regression models included age, history of fracture and all covariates that were univariately associated with the risk of fractures and significantly contributed to the multivariate model, i.e. inclusion of the covariate resulted in a change of the compliance hazard ratio (HR) of $5 \%$ or over, starting with the most potent covariate. HRs and $95 \%$ confidence intervals (CI) were estimated using SAS V8.2 UNIX (Cary, NC, USA).

\section{Results}

The study cohort included 8,822 new female users of daily or weekly alendronate or risedronate, who contributed a total of 22,484 person-years of follow-up during the study period. More than half of the patients were older than 70 years of age $(n=4,708,53 \%)$ and about one quarter of the patients used corticosteroids (irrespective of quantity) (Table 1). The first bisphosphonate was mainly prescribed by the general practitioner (64\%). Only 197 patients (2\%) were hospitalized for an osteoporotic fracture in the year before starting treatment.

During follow-up, 216 patients (2\%) experienced an osteoporotic fracture, of which 40 patients during the first six months and 78 during the first year. About two third of all osteoporotic fractures were located at the proximal femur.

The percentage of patients with a MPR $<80 \%$ (i.e. noncompliant patients) increased from $34 \%(3,018 / 8,822)$ after six months of follow-up to $42 \%(3,743 / 8,822)$ after one year, $51 \%(3,014 / 5,883)$ after two years and $60 \%(2,149 / 3,615)$ after three years of follow-up. Subdividing MPR in classes (Fig. 1), the majority of patients either had a MPR $\geq 90 \%$ or a MPR $<20 \%$, with the first group decreasing and the latter group increasing over time.

Dichotomizing compliance, a MPR $<80 \%$, i.e. noncompliant bisphosphonate use was associated with a $40 \%$ increased risk of osteoporotic fracture more than six months

Table 1 General characteristics of new bisphosphonate users between 1 January 1999 and 30 June $2004(N=8,822)$

\begin{tabular}{|c|c|c|c|}
\hline Characteristic & & $\mathrm{N}$ & $\%$ \\
\hline \multirow[t]{3}{*}{ Age class (years) } & $45-54$ & 889 & 10.1 \\
\hline & $55-69$ & 3,225 & 36.5 \\
\hline & $\geq 70$ & 4,708 & 53.4 \\
\hline \multicolumn{2}{|l|}{ Mean age (years) \pm sd } & $69.4 \pm 10.3$ & \\
\hline \multirow[t]{3}{*}{ Year of start } & $1999 / 2000$ & 2,250 & 25.5 \\
\hline & $2001 / 2002$ & 2,769 & 31.4 \\
\hline & $2003 / 2004$ & 3,803 & 43.1 \\
\hline \multirow[t]{2}{*}{ Initial bisphosphonate } & Daily bisphosphonate & 4,222 & 47.9 \\
\hline & Weekly bisphosphonate & 4,600 & 52.1 \\
\hline \multirow[t]{3}{*}{ First prescriber } & General practitioner & 5,704 & 64.7 \\
\hline & Internist, rheumatologist, orthopedist & 2,313 & 26.2 \\
\hline & Other prescribers & 805 & 9.1 \\
\hline \multirow{2}{*}{\multicolumn{2}{|c|}{$\begin{array}{l}\text { Hospitalization for an osteoporotic fracture in the year before start } \\
\text { Use of corticosteroids in the year before start (irrespective of quantity) }\end{array}$}} & 197 & 2.2 \\
\hline & & 2,389 & 27.1 \\
\hline \multirow[t]{3}{*}{ Follow-up time (months) } & $12-23$ & 2,939 & 33.3 \\
\hline & $24-35$ & 2,268 & 25.7 \\
\hline & $\geq 36$ & 3,615 & 41.0 \\
\hline
\end{tabular}




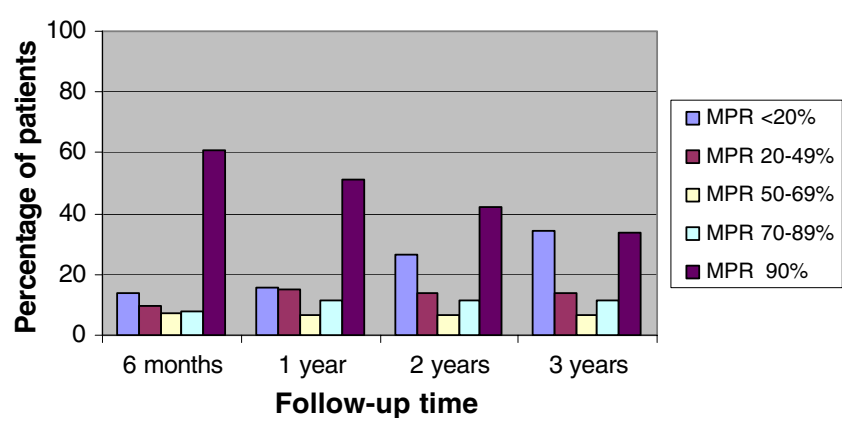

Fig. 1 Compliance with bisphosphonates, classified in five MPR categories, after six months, one year, two years and three years of follow-up. MPR: medication possession ratio

after starting treatment (HR 1.41 95\% CI 1.04-1.91, adjusted for age and history of fracture (see Table 2 for association between co-variates and fracture risk)) compared to a MPR $\geq 80 \%$, i.e. compliant use. Varying the exclusion period, i.e. excluding fractures occurring in the first year of follow-up or including all fractures during follow-up, yielded similar adjusted increased risks (HR 1.50; 95\%CI 1.06-2.13 and HR 1.45; 95\%CI 1.10-1.91, respectively). Excluding women who received only one bisphosphonate dispensing during follow-up also did not change the results (adjusted HR 1.45; 95\% CI 1.05-1.99, based on 156 fracture patients and 7,758 fracture-free patients).

Classifying compliance into five categories, fracture risk gradually increased with poorer compliance (p-value $<0.05$ for trend). Compared to a MPR $\geq 90 \%$, the adjusted risk of fracture increased from 1.2 times for a MPR between $50 \%$ and $90 \%$ to 1.8 times for a MPR less than $20 \%$ (Fig. 2).

\section{Discussion}

This study indicates that non-compliant bisphosphonate users had an approximately 50\% higher likelihood of osteoporotic fracture compared to compliant users. Classifying compliance into five categories, fracture risk gradually increased with poorer compliance to an $80 \%$ risk increase with very low compliance compared to very high compliance. These results emphasize the importance of treatment compliance in obtaining maximal treatment benefit.

To quantify the loss of treatment benefit with low compliance, information on fracture risk in women not treated with bisphosphonates is necessary. The above mentioned percentage risk increases are compared to compliant use and cannot be translated into percentage loss of treatment benefit. We chose not to use untreated patients as reference because of the heterogeneity of patterns of treatment use that exist within this group. In a meta-analysis of 11 trials of alendronate for the treatment of postmenopausal osteoporosis, a consistent $50 \%$ reduction in fracture risk was seen compared to placebo [18]. For risedronate, a meta-analysis of 8 trials revealed a 25 to $35 \%$ reduction in fracture risk compared to placebo [19]. In other words, untreated women are 1.5 to 2 times as likely to experience a fracture compared to women using bisphosphonates.

Table 2 Association between co-variates and fracture risk more than six months after starting treatment

\begin{tabular}{|c|c|c|c|c|c|c|}
\hline & \multicolumn{2}{|c|}{ Fracture patients $(\mathrm{N})$} & \multicolumn{2}{|c|}{ Fracture-free patients $(\mathrm{N})$} & \multirow[b]{2}{*}{$\mathrm{HR}_{\text {crude }}$} & \multirow[b]{2}{*}{$95 \% \mathrm{CI}$} \\
\hline & $\mathrm{N}$ & $\%$ & $\mathrm{~N}$ & $\%$ & & \\
\hline Total & 176 & 100 & 8,606 & 100 & & \\
\hline \multicolumn{7}{|l|}{ Age (years) at start } \\
\hline $45-54$ & 7 & 4.0 & 879 & 10.2 & 1.00 & reference \\
\hline $55-69$ & 29 & 16.5 & 3,189 & 37.1 & 1.16 & $0.51-2.66$ \\
\hline$\geq 70$ & 140 & 79.5 & 4,538 & 52.7 & 4.28 & $2.00-9.15$ \\
\hline \multicolumn{7}{|l|}{ Year of start } \\
\hline $1999 / 2000$ & 86 & 48.9 & 2,158 & 25.1 & 1.68 & $1.04-2.71$ \\
\hline $2001 / 2002$ & 63 & 35.8 & 2,691 & 31.3 & 1.55 & $0.97-2.49$ \\
\hline $2003 / 2004$ & 27 & 15.3 & 3,757 & 43.6 & 1.00 & reference \\
\hline History of osteoporotic fracture & 5 & 2.8 & 190 & 2.2 & 1.52 & $0.63-3.70$ \\
\hline \multicolumn{7}{|c|}{ Comedication in the year after start ${ }^{1}$} \\
\hline Analgesics & 68 & 38.6 & 2,136 & 24.8 & 1.82 & $1.34-2.46$ \\
\hline Benzodiazepines & 80 & 45.5 & 3,324 & 38.6 & 1.30 & $0.96-1.74$ \\
\hline Antidepressants & 29 & 16.5 & 1,046 & 12.2 & 1.52 & $1.02-2.26$ \\
\hline ß-blockers & 47 & 26.7 & 1,929 & 22.4 & 1.35 & $0.97-1.89$ \\
\hline
\end{tabular}

HR: hazard ratio, CI: confidence interval

${ }^{1}$ Univariate non-significant comedications (i.e. non-steroidal anti-inflammatory drugs, thiazides and statins) are not shown. 


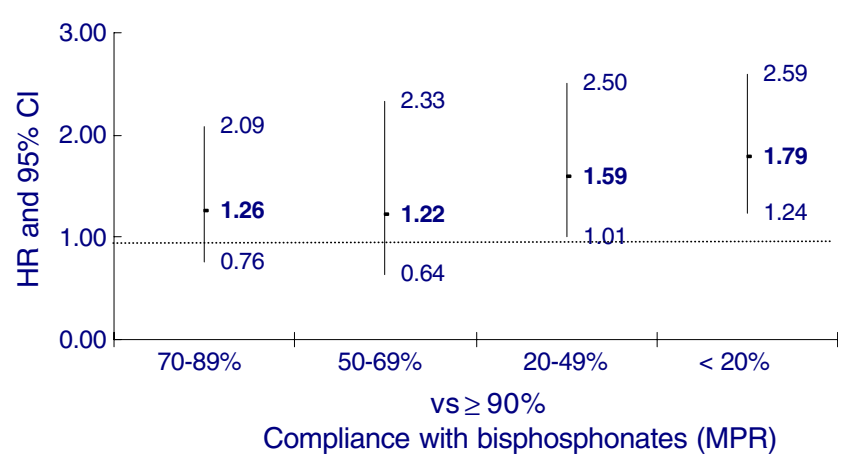

Fig. 2 The relationship between compliance with bisphosphonates, classified in five MPR categories, and fracture risk more than half a year after starting treatment. HRs are adjusted for age and history of fracture and are compared to a MPR $\geq 90 \%$. MPR: medication possession ratio, HR: hazard ratio, CI: confidence interval

Assuming that the patients with a MPR $\geq 90 \%$ in our study are comparable to the treated patients in trials, the 1.6 to 1.8 times increased fracture risk we observed with a MPR $<50 \%$ can be translated into a 80 to $100 \%$ loss of treatment benefit. Apparently, patients with the lowest compliance are essentially comparable to non-treated patients.

At present, in addition to Caro et al., others have reported on the relationship between compliance with bisphosphonates and fracture risk [11, 20,21]. Siris et al. [20] found a relative fracture risk reduction of $25 \%$ with compliant use of alendronate or risedronate, i.e. a $33 \%$ risk increase with non-compliant use. There was a progressive relationship between compliance and fracture risk reduction, commencing at a MPR around 50\% and becoming more pronounced at a MPR $\geq 75 \%$. This finding, like our results, suggests that low-compliant patients gain only marginal benefit from bisphosphonate therapy. Among women starting drug therapy for osteoporosis (of which two-third bisphosphonates) Weycker et al. [21] found that compared to women with a MPR $<30 \%$, the odds of fracture was similar (1.02) for women with a MPR between 30 and $69 \%$ and women with a MPR between 70 and $89 \%$, but lower (0.70) for women with a MPR $\geq 90 \%$. The latter corresponds to a 1.4 times increased risk of fracture with a MPR $<30 \%$ compared to a MPR $\geq 90 \%$. Caro et al. [11] reported that compared to a MPR $>90 \%$, fracture risk was significantly increased at lower compliance levels, i.e. up to 1.4 times with a MPR $\leq 50 \%$. However, it is not clear whether this effect was gradual. In a study population of mainly users of hormone replacement therapy, Huybrechts et al. [22] found that compared to women with a MPR $\geq 90 \%$, fracture risk was similar (HR 1.09) for women with a MPR between 80 and $90 \%$, but higher for women with a MPR between 50 and $80 \%$ and women with a MPR $<50 \%$ (HR 1.18 and 1.21, respectively). Our associations are about similar than reported by Siris et al., but stronger than found by Caro et al., Weycker et al. and Huybrechts et al.
The most likely explanation for this is that our and Siris' study population was restricted to bisphosphonate users, while the others also included users of other, less potent antiosteoporotic drugs (30\% to $65 \%$ of the study population).

There is a previous PHARMO study on bisphosphonate use and risk of fractures, using persistence [5]. Besides a different measure of drug exposure, the current study differs from this previous study in that the study period was extended, different inclusion criteria were applied and new patients were included besides previously studied patients. Although persistence and compliance are measured differently, with persistence referring to the duration of uninterrupted drug use, they are closely related. Patients classified as non-compliant would also have been classified as nonpersistent and vice versa. The results of our current study are in line with our previous study in which the risk of fractures was reduced by $26 \%$ after one year persistent bisphosphonate use and up to $32 \%$ after two years of persistent use [5]. Similarly, Gold et al [23] reported that persistent bisphosphonate use for at least 6 months was associated with a 26\% lower fracture risk. Expressing these results the other way around, non-persistent bisphosphonate use is associated with an about $40 \%$ fracture risk increase. Among a population of mainly users of hormone replacement therapy, one year of uninterrupted therapy reduced the likelihood of fractures with significant odds ratios of 0.38 for hip fractures and 0.60 for vertebral fractures [7].

Our analyses may be limited by some issues common to observational database research in daily clinical practice. Most importantly, although we considered a large number of known confounders in our analyses, including history of fractures, there may be other confounding factors for which information was not available, such as bone mineral density (BMD). BMD is an important determinant of fracture risk [24] and BMD testing has been shown to be positively associated with persistence and compliance $[25,26]$. Consequently, the results of our analyses are likely to be conservative in the sense that compliant patients may have had an increased fracture risk because of low BMD, and history of fractures only partially controlled for this. Furthermore, it is possible that patients have started using other anti-osteoporotic drugs, e.g. raloxifene, during follow-up and stopped bisphosphonate use. This means that part of the patients who were non-compliant with bisphosphonates may have been protected against fractures by another anti-osteoporotic drug and the observed increased fracture risk with non-compliant bisphosphonate use therefore may be underestimated. However, we know from our database that this concerns only about $7 \%$ of patients in the first year of bisphosphonate treatment. In addition, compliance with treatment was based on dispensing data. As it is unknown whether a patient actually used the drug, compliance may have been overestimated. 
However, it seems likely that patients who obtain prescription refills do take their medication. Excluding women who filled only one bisphosphonate dispensing during follow-up from the analyses did not change the results. Another limitation relates to the classified analysis. With the use of five compliance categories studying a trend is possible but it does not give information on the point at which there is a significant shift in fracture risk. Ideally, 10\% MPR categories should be used. However, patient numbers per $10 \%$ MPR categories (data not shown) did not allow for this detailed analysis.

To increase the treatment benefit of bisphosphonates, compliance should be improved. Related to the inconvenient, stringent intake regimen, dosing frequency is an important determinant of compliance with bisphosphonates. In recent earlier studies, once-weekly dosing was associated with better compliance and persistence compared to daily dosing. However, even with the weekly regimen, compliance and persistence were suboptimal in about half of the patients $[6,8,9]$. Bisphosphonates with less frequent dosing regimes than weekly, e.g. monthly or three-monthly ibandronate or annual zolendronate, are either available yet or upcoming and may further improve compliance and therefore treatment benefit. In a cross-over trial, women with postmenopausal osteoporosis preferred once-monthly ibandronate therapy and found it more convenient than once-weekly alendronate therapy [27]. There are no data yet on the actual impact of this once-monthly, and other formulations on compliance with bisphosphonate therapy and reduction of fracture risk in daily practice. Other keys to improve compliance are e.g. healthcare provider - patient communication and continuous reinforcement of the importance of treatment. Reminder systems may help the patient comply with therapy, especially with infrequent dosing regimes. However, results from a review show that current methods of improving patient compliance for several chronic health problems are mostly not very successful [28]. Furthermore, although a number of demographic and clinical variables are associated with compliance with bisphosphonates these predictors, either individually or together, have been shown to have low predictive value for identifying patients who will become non-compliant [25].

In conclusion, the results of this study show a statistically significant association between level of compliance with bisphosphonates and level of fracture risk, emphasizing the importance of treatment compliance in obtaining maximal bone protection.

Acknowledgement The study was financially supported by an unrestricted grant from Novartis Pharma AG, Basel, Switzerland. We gratefully acknowledge the critical review of the study by A.M.T. Engbersen from Novartis Pharma AG, Basel, Switzerland.

\section{References}

1. NIH Consensus Development Panel on Osteoporosis Prevention, Diagnosis, and Therapy (2001) Osteoporosis prevention, diagnosis, and therapy. JAMA 285:785-795

2. Cummings SR, Melton LJ (2002) Epidemiology and outcomes of osteoporotic fractures. Lancet 359:1761-1767

3. McClung MR (2000) Bisphosphonates in osteoporosis: recent clinical experience. Expert Opin Pharmacother 1:225-238

4. Bone HG, Hosking D, Devogelaer JP, et al. (2004) Ten years' experience with alendronate for osteoporosis in postmenopausal women. N Engl J Med 350:1189-1199

5. van den Boogaard CHA, Breekveldt- Postma NS, Borggreve SE, et al.(2006) Persistent bisphosphonate use and the risk of osteoporotic fractures in clinical practice: a database analysis study. Curr Med Res Opin 22:1757-1764

6. Cramer JA, Amonkar MM, Hebborn A, et al. (2005) Compliance and persistence with bisphosphonate dosing regimens among women with postmenopausal osteoporosis. Curr Med Res Opin 21:1453-1460

7. McCombs JS, Thiebaud P, McLaughlin-Miley C, et al. (2004) Compliance with drug therapies for the treatment and prevention of osteoporosis. Maturitas 48:271-287

8. Penning-van Beest FJA, Goettsch WG, Erkens JA, et al. (2006) Determinants of persistence with bisphosphonates: a study in women with postmenopausal osteoporosis. Clin Ther 28:236-242

9. Recker RR, Gallagher R, MacCosbe PE (2005) Effect of dosing frequency on bisphosphonate medication adherence in a large longitudinal cohort of women. Mayo Clin Proc 80:856-861

10. Baker DE (2002) Alendronate and risedronate: what you need to know about their upper gastrointestinal tract toxicity. Rev Gastroenterol Disord 2:20-33

11. Caro JJ, Ishak KJ, Huybrechts KF, et al. (2004) The impact of compliance with osteoporosis therapy on fracture rates in actual practice. Osteoporos Int 15:1003-1008

12. Hubbard R, Farrington P, Smith C, et al. (2003) Exposure to tricyclic and selective serotonin reuptake inhibitor antidepressants and the risk of hip fracture. Am J Epidemiol 158:77-84

13. van Staa TP, Leufkens HGM, Cooper C (2002) Utility of medical and drug history in fracture risk prediction among men and women. Bone 31:508-514

14. Herings RM, Stricker BH, de Boer A, et al. (1995) Benzodiazepines and the risk of falling leading to femur fractures. Dosage more important than elimination half-life. Arch Intern Med 155:1801-1807

15. Pasco JA, Kotowicz MA, Henry MJ, et al. (2002) Statin use, bone mineral density, and fracture risk: Geelong Osteoporosis Study. Arch Intern Med 162:537-540

16. Schlienger RG, Kraenzlin ME, Jick SS, et al. (2004) Use of betablockers and risk of fractures. JAMA 292:1326-1332

17. Herings RM, Stricker BH, de Boer A, et al. (1996) Current use of thiazide diuretics and prevention of femur fractures. J Clin Epidemiol 49:115-119

18. Cranney A, Wells G, Willan A, et al. (2002) Meta-analyses of therapies for postmenopausal osteoporosis. II. Meta-analysis of alendronate for the treatment of postmenopausal women. Endocr Rev 23:508-516

19. Cranney A, Tugwell P, Adachi J, et al. (2002) Meta-analyses of therapies for postmenopausal osteoporosis. III. Meta-analysis of risedronate for the treatment of postmenopausal osteoporosis. Endocr Rev 23:517-523

20. Siris ES, Harris ST, Rosen CJ, et al. (2006) Adherence to bisphosphonate therapy and fracture rates in osteoporotic women: relationship to vertebral and nonvertebral fractures from 2 US claims databases. Mayo Clin Proc 81:1013-1022 
21. Weycker D, Macarios D, Edelsberg J, et al. (2007) Compliance with osteoporosis drug therapy and risk of fracture. Osteoporos Int 18:271-277

22. Huybrechts KF, Ishak KJ, Caro JJ (2006) Assessment of compliance with osteoporosis treatment and its consequences in a managed care population. Bone 38:922-928.

23. Gold, DT, Martin BC, Frytak JR, et al. (2007) A claims database analysis of persistence with alendronate therapy and fracture risk in post-menopausal women with osteoporosis. Curr Med Res Opin 23:585-594

24. Johnell O, Kanis JA, Oden A, et al. (2005) Predictive value of BMD for hip and other fractures. J Bone Miner Res 20:1185-1194
25. Lo JC, Pressman AR, Omar MA, et al. (2006) Persistence with weekly alendronate therapy among postmenopausal women. Osteoporos Int 17:922-928

26. Solomon DH, Avorn J, Katz JN, et al. (2005) Compliance with osteoporosis medications. Arch Intern Med 165:2414-2419

27. Emkey R, Koltun W, Beusterien K, et al. (2005) Patient preference for once-monthly ibandronate versus once-weekly alendronate in a randomized, open-label, cross-over trial: the Boniva Alendronate Trial in Osteoporosis (BALTO). Curr Med Res Opin 21:1895-1903

28. McDonald HP, Garg AX, Haynes RB (2002) Interventions to enhance patient adherence to medication prescriptions: scientific review. JAMA 288:2868-2879 